\title{
PRELIMINARY EVALUATION OF A COMMERCIAL 360 MULTI-CAMERA RIG FOR PHOTOGRAMMETRIC PURPOSES
}

\author{
L. Teppati Losè ${ }^{1 *}$, F. Chiabrando ${ }^{1}$, A. Spanò ${ }^{1}$ \\ ${ }^{1}$ Politecnico di Torino, Dept. of Architecture and Design - Geomatics for Cultural Heritage Laboratory (LabG4CH) \\ (lorenzo.teppati, filiberto.chiabrando, antonia.spano)@polito.it
}

Commission II, WG II/8

KEY WORDS: Calibration, 360 Images, Multi-Camera system, Cultural Heritage, Photogrammetry

\begin{abstract}
:
The research presented in this paper is focused on a preliminary evaluation of a 360 multi-camera rig: the possibilities to use the images acquired by the system in a photogrammetric workflow and for the creation of spherical images are investigated and different tests and analyses are reported. Particular attention is dedicated to different operative approaches for the estimation of the interior orientation parameters of the cameras, both from an operative and theoretical point of view. The consistency of the six cameras that compose the 360 system was in depth analysed adopting a self-calibration approach in a commercial photogrammetric software solution. A 3D calibration field was projected and created, and several topographic measurements were performed in order to have a set of control points to enhance and control the photogrammetric process. The influence of the interior parameters of the six cameras were analyse both in the different phases of the photogrammetric workflow (reprojection errors on the single tie point, dense cloud generation, geometrical description of the surveyed object, etc.), both in the stitching of the different images into a single spherical panorama (some consideration on the influence of the camera parameters on the overall quality of the spherical image are reported also in these section).
\end{abstract}

\section{INTRODUCTION}

In recent years, the commercial world of entertainment (that is constantly and rapidly changing) has gone through some new major transformations, both from the customers side and from the major companies involved in the market. More specifically, the biggest changes can be identified in the segment related to the production and sharing of multimedia immersive contents, e.g. the 360 images and videos. Researchers in the field of Geomatics have monitored the evolution of the market, in order to stress the possibility of using these new systems for metric documentation purposes, with the typical adopted methodologies of their discipline.

\subsection{Rise of 360 multi-cameras systems}

Different technological observers have defined 2017 as the year of 360 cameras and for several reasons this affirmation can be considered true: the past year brought important updates, the launch of several new cameras with different range of prices and some major technological improvements. Consequently, related to the new diffusion of sensors, the development or implementation of new platforms for the use and diffusion of this contents is growing as well. However, as is well known, this is not a brand-new technique: the idea of combining together different images to create wide format omni-comprehensive representation of the physical space dates back to the late XIX century and has constantly evolved during time. Also, the use of these panoramic images for photogrammetric purposes has a history that dates back in time (Luhmann, 2004) .

Single and multi-camera systems devoted to the immersive recording of the physical space were already developed and used before this recent technological acceleration, also thanks to the implementation of new algorithms for the image stitching and the massive use of digital technologies (lot of these improvement can be derived from the computer disciplines as reporte in Szeliski, 2006). The major and most interesting last advancements in this technique are related with the development of ad hoc systems and software with reduced cost and with the possibility to grant a wider range of people the accessibility to this kind of technology.

\subsection{Action and 360 cameras in photogrammetry}

Among the different COTS (Commercial Off The Shelf) sensors available two categories have been particularly investigated by Geomatics researchers in the last years: action cameras and 360 cameras. The use of action cameras for photogrammetric purposes have been investigated by a large number of researchers in the last years (Balletti, Guerra, Tsioukas, \& Vernier, 2014; Barazzetti, Previtali, \& Roncoroni, 2017b; Markiewicz, Lapi, Bienkowski, \& Kaliszewska, 2017; Perfetti, Polari, \& Fassi, 2017) and several issues related with these sensors have been considered: fisheye lenses distortion, short focal lens influence, mathematical model adopted for the calibration, etc.

On the other hand, the research on 360 cameras has grown in interest recently, due to the release on the market of new and lowcost sensors. More complex and expensive systems, e.g. Ladybug by FLIR, the 360 camera of Google by Immersive Media, iSTAR by NCTech or images composed by DSRL (Digital single lens reflex) wide angle cameras, already existed and have been used but the availability of new less expensive platforms, gave new launch to the research in this sector. In this case, the attention of the researchers (Barazzetti, Previtali, \& Roncoroni, 2017a; Fangi, 2015; Fangi \& Nardinocchi, 2013; Holdener, Nebiker, \& Blaser, 2017; Kossieris, Kourounioti, Agrafiotis, \& Georgopoulos, 2017) was focused on the issues related with the system configuration, the stitching of images, the different approaches to use compared with traditional photogrammetry, etc.

\subsection{The tested system}

In the research presented in this contribute a commercial system, that combine the research on action and 360 camera, was tested: the Freedom 360 (www. freedom360.us). The system (Figure 1) is composed by a $3 \mathrm{D}$ printed mount design to hold six action cameras (GoPro Hero4 or Hero3) coupled in opposite positions to record full spherical immersive videos or images. The main attractive features

\footnotetext{
* Corresponding author
} 
of this system are related to different characteristics: the relative low cost of the Freedom 360 compared with other similar systems, its portability (size less than $10 \times 10 \times 10 \mathrm{~cm}$ and total weight with cameras of $525 \mathrm{~g}$ ), the possibility of controlling individually and independently the six cameras and consequently to have the chance to manage and process the data recorded from the six cameras both separately or together.

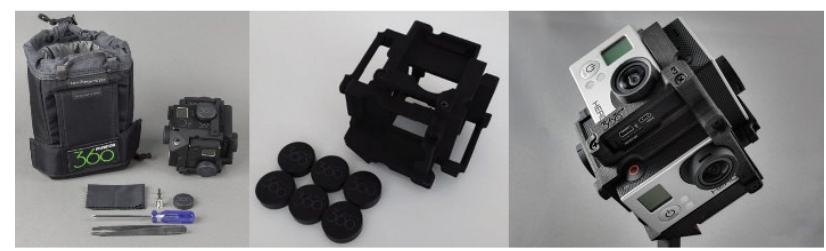

Figure 1. The Freedom 360 system.

There are obviously some drawbacks: the acquisition and processing of the data collected by this system of sensors is less controlled, compared to other more expensive solutions, and is not always easy to reach good results during the creation of 360 contents. Moreover, the overall quality of the system is directly influenced by the characteristics of the single camera. Due to the reasons mentioned above and to the intrinsic characteristics of the system one of the main aim of the presented research is to assess and verify the consistency of the six action cameras, and consequently of the system itself, before performing some tests in the contest of the documentation of a real Built Heritage environment.

\section{THE PROPOSED METHODOLOGY}

The first tests were carried out in order to verify the consistency of the six cameras: as is well documented by other works (Balletti et al., 2014; Barazzetti et al., 2017b; Läbe \& Förstner, 2004), low cost commercial cameras are equipped with less stable lenses and the camera parameters need to be carefully considered. These issues need to be in depth investigated and can have an impact on different aspects related with the use of this kind of system. The parameters of the camera's sensors can be useful in the generation of good quality panoramic images, derived from the stitching of different acquisitions together and, as is well known (Fraser, 2013; Luhmann, Fraser, \& Maas, 2016; Schneider, Schwalbe, \& Maas, 2009), in case of photogrammetric approaches they are crucial (in order to achieve more reliable and metric controlled results).

\subsection{Employed system and 3D calibration environment}

For our test the Freedom 360 was equipped with six GoPro Hero 4 Silver Edition, main specifications of the camera are reported in Table 1. GoPro Hero 4 Silver main specifications

\begin{tabular}{|l|l|}
\hline Weight & $84 \mathrm{gr}$ \\
\hline Size & $54 \times 41 \times 30 \mathrm{~mm}$ \\
\hline Sensor & $\mathrm{CMOS}-12 \mathrm{MP}$ \\
\hline Sensor size & $1 / 2.3^{\prime \prime}$ \\
\hline Focal length & $2.92 \mathrm{~mm}$ \\
\hline Image resolution & Max $4000 \times 3000$ \\
\hline
\end{tabular}

Table 1. GoPro Hero 4 Silver main specifications.

To estimate and evaluate these factors different tests for the calibration of the cameras were carried out. Then, a specific 3D calibration field was projected and realised (Figure 2, a). The 3D field was created taking into consideration different factors: presenting a marked 3D component with objects at different depth, having recognizable features to be used in the photogrammetric process, simulate both an indoor/outdoor environment with a short (and as much constant as possible) acquisition distance. On the calibration field a set of targets was homogenously distributed in order to use the $3 \mathrm{D}$ coordinates of that measured points for the calibration process. The 14 targets were measured using a typical traditional approach: two points forward intersection in order to obtain with a good accuracy the coordinates of the points. For the topographic survey a Leica Viva TS16 Total Station was used: accuracy of 1" (0.3 mgon) on angular measurement and distance accuracy on prism of $1 \mathrm{~mm}+1.5$ parts per million. In order to obtain the best accuracy and precision as possible in the measurement of the distance a circular mini-prism were used during the topographic survey.

The data collected on the field were then adjusted using MicroSurvey STAR*NET software (Figure 2, b) where the planimetric and altimetric components of the forward intersection were separately considered. According to the acquisition geometry, distances and adopted strategy the residual on the 14 targets for both the components planimetric and altimetric is less than $2 \mathrm{~mm}$. The final 3D coordinates were then used in the photogrammetric approach both as GCPs (Ground Control Points) and CPs (Check Points) to precisely estimate and control the camera interior parameters and to scale and georeference the generated models.

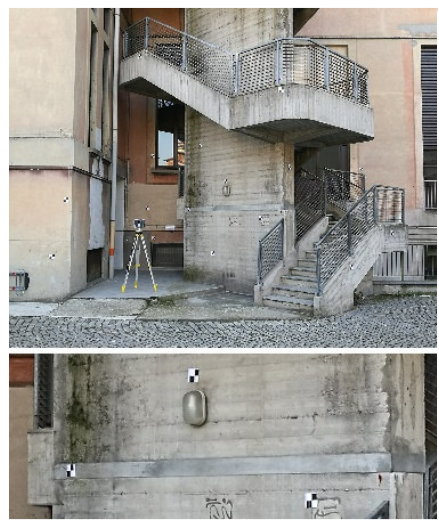

(a)

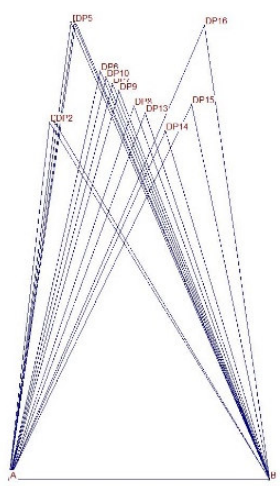

(b)
Figure 2. The calibration field (a); top view of the compensated topographic network (b).

\subsection{Proposed approach and acquisitions}

In order to verify the consistency of the system and estimate the interior orientation parameters of each one of the cameras, the six GoPro were marked with an identification letter and different photogrammetric acquisition were performed:

Each one of the six cameras was detached from the rig and inserted on an ad hoc realized 3D printed support in order to mount it on a photographic tripod for image acquisition. With this configuration, a dense set of images was acquired for each camera (with different camera orientation and relative position). An average number of 150 images were obtained for each of the six cameras.

- All the six cameras were mounted on the Freedom 360 rig and used in the time-controlled modality for the shoot. The system was then moved in different preselected positions to acquire the whole calibration field. The images were then selected and only the images acquired in the chosen positions were considered. A total of 258 images were acquired with the 360 configuration, corresponding to 43 preselected position of the rig.

An example of the two networks of images acquired is reported below in Figure 3. 


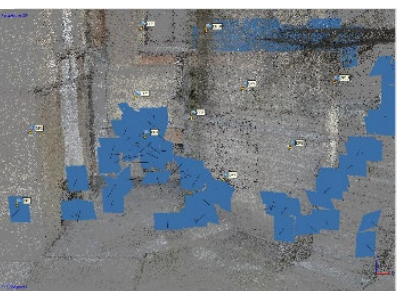

(a)

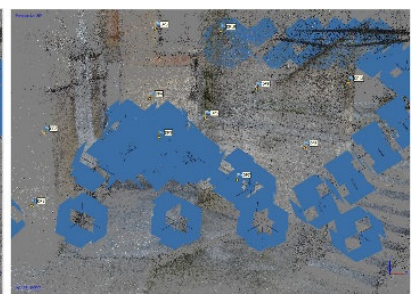

(b)
Figure 3. Part of the images network acquired on the calibration field. One of the six camera (a) and the six cameras mounted on the 360 rig (b).

\subsection{Interior orientation parameters estimation}

In the presented research a self-calibration approach (Fraser, 1997; Gruen \& Beyer, 2001; Remondino \& Fraser, 2006) was adopted in order to estimate the interior orientation parameters of every camera and analyse their consistency, using a wellknown commercial software solution for SfM (Structure from Motion): Agisoft Photoscan.

The above-mentioned acquisitions were processed in eight different projects, divided as following:

- Six individual projects (one for each action camera mounted on the photographic tripod).

- One project for the images acquired with the 360 rig, using the native Exif (Exchangeable image file format) metadata embedded in the cameras.

- $\quad$ One last project for the images acquired with the 360 rig, but applying a modification on the Exif metadata, in order to have the software recognizing the six cameras as different.

For each project 8 of the measured target were used as GCPs while 6 were used as CPs, the RMSe (Root Mean Square error) on these points is reported in the following Figure 4.

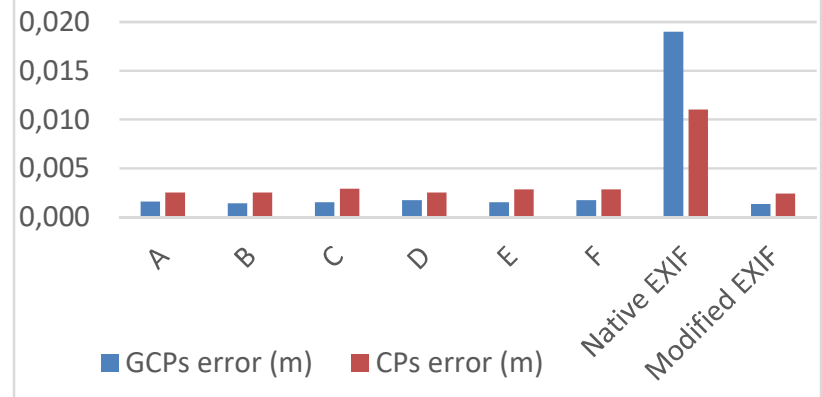

Figure 4. RMSe on GCPs and CPs of the different photogrammetric projects.

As will be discussed in paragraph 2.3.2, adopting the automated solution, i.e. using a single calibration model for the six cameras, will produce poor results in terms of metric accuracy as well as in the overall quality of the generated 3D model.

Furthermore, all the projects were processed using the same parameters for the estimation of interior and exterior orientation, tie point extraction and BBA (Bundle Block Adjustment): the accuracy of the alignment was set as high in order to estimate at least three coefficients for the radial distortion and two for the tangential, plus the focal length, the principal point coordinates and the skew transformation coefficients. Key/tie points limit was set at 0 in order to extract as many points as possible.

\subsubsection{Interior orientation evaluation using Six cameras separately considered}

The six action cameras of the 360 system were previously marked with letters (A, B, C, D, E, F) to create an unique identification of each GoPro and of their reciprocal positions on the rig. The GCPs and CPs were placed in all the six projects and the first steps of the photogrammetric process were completed. After the estimation of the interior orientation parameters for each camera, the data were collected and organize in order to be compared and analysed. The estimated parameters of the six cameras are reported in Table 2 (Appendix): focal length in pixels and millimetres (f), principal point coordinates in pixels (cx and cy), radial distortion coefficients in millimetres $(\mathrm{k} 1, \mathrm{k} 2, \mathrm{k} 3)$, skew coefficients in pixels (b1 and b2) and tangential distortion coefficients in millimetres ( $\mathrm{p} 1$ and $\mathrm{p} 2$ ).

Especially in the case of these low-cost mass market sensors these parameters need to be carefully considered.

In this case the estimated focal length of the six cameras can be considered consistent and similar, while some issues can be traced in the estimation of the principal point coordinates of the different sensors, i.e. coordinates of lens optical axis interception with sensor plane (expressed in pixels with $\mathrm{cx}$ and cy coefficients). The estimated principal points of the six cameras sensors is graphically represented in the following Figure 5.

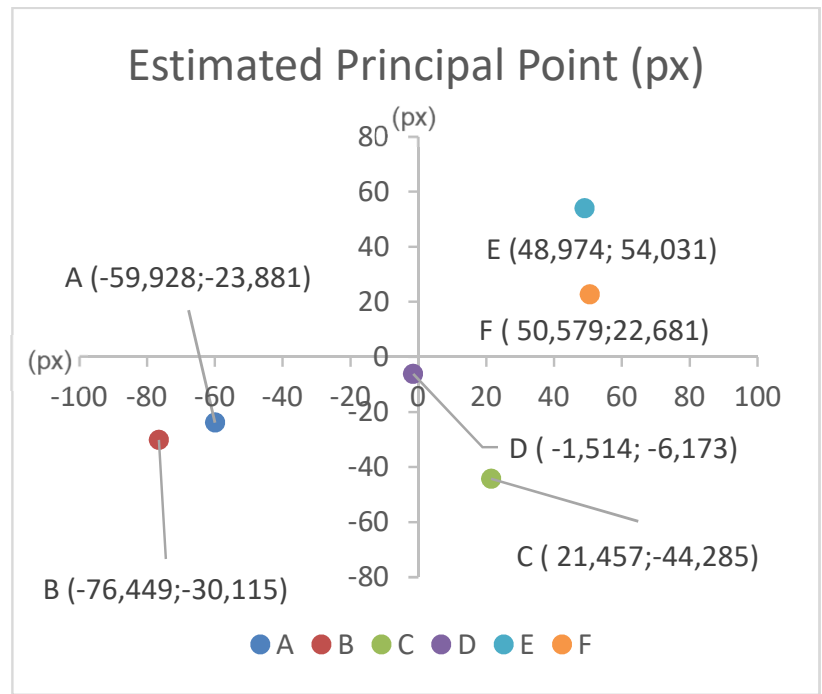

Figure 5. Estimated principal point coordinates of the six cameras separately considered and processed.

As is possible to notice, the principal point coordinates of five out of six cameras can be considered comparable (the deviation from the ideal principal point of coordinates 0,0 has the same order of magnitude but located on different quarter of the sensor) while the camera $\mathrm{D}$ presents a complete different position, almost in the ideal intersection of the two principal axes. This camera, which value is theoretically closer to the ideal principal point, can create some issues when working together with the other five sensors, due to its different interior parameters. As we will see, this can be considered an issue both when working with the stitching of the six images in a single spherical image, both in the photogrammetric process.

\subsubsection{Six cameras mounted on the rig and automatically processed}

The images acquired by the six cameras mounted on the rig were processed both following the automatic workflow implemented in Photoscan, and in a second time with a manual editing to better control the camera parameters (paragraph 2.3.3). During the 
automatic workflow, the software uses the information derived from the Exif metadata of the six cameras as initial internal orientation for the further camera parameters estimation. In this case, due to the information embedded in the Exif, Photoscan assumes that only a camera was used and perform all the phases of the photogrammetric process considering all the cameras as identical. This wrong assumption lead to different problems in the phases of interior orientation parameters estimation and tie point extraction. These issues are evident for example in the value computed for GCPs and CPs errors (Figure 4). Also, in this case is interesting to analyse the values estimated for the principal point coordinates (Figure 6) that are similar to the ones of camera $\mathrm{D}$, presented in the previous paragraph. The values of the deviation of the six different cameras from the principal point coordinates that were evident from the previous estimations are not considered in this case.

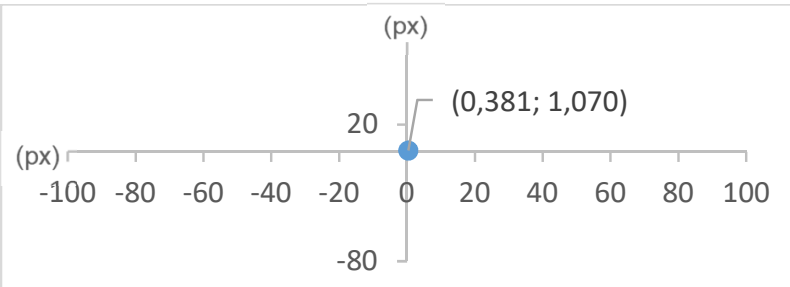

- Rig_Native EXIF

Figure 6. Estimated principal point coordinates of the six cameras mounted on the rig and automatically processed with the native Exif information.

The issues derived from this approach are also clearly visible in another representation: the plotting of the image residuals reported below in Figure 7.

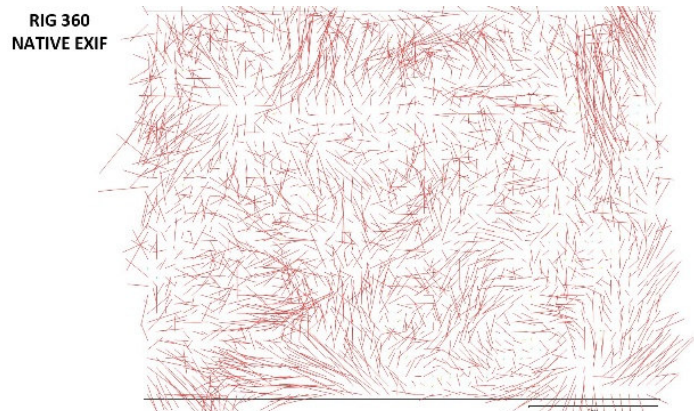

Figure 7. Image residuals for the 360 configuration automatically processed with the native Exif.

In this case is clearly shown that the interior parameters estimated for the camera are not generating consistent results and that performing a single calibration for the six cameras, following an automatic approach, is not a successful and satisfying solution. The other estimated parameters of the interior orientation are reported in Table 3.

\subsubsection{Interior orientation evaluation using Six cameras} mounted on the rig and processed after Exif modification

To evaluate if it was possible to contemporary calibrate, and with satisfying result, the six cameras mounted on the rig another project was created and processed. Before importing the images in the software, the information embedded in the Exif files were modified and the name of the camera model was changed, in order to process independently the six cameras during the workflow. The estimated interior parameters are reported in Table 4, while the estimated coordinates of the six principal points are shown in the images below, Figure 8 .

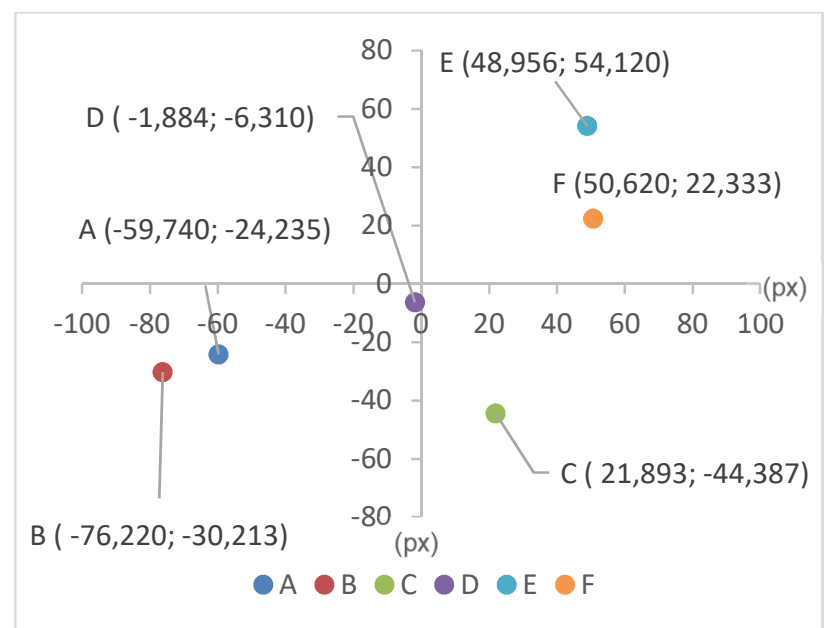

Figure 8. Estimated principal point coordinates of the six cameras mounted on the rig and processed after the Exif modification.

As shown in Figure 4, adopting this approach is possible to obtain an RMSe on the GCPs and CPs that can be compared with the one achieved while working with the six cameras independently.

\section{ANALISYS OF THE RESULTS}

The data obtained by the different tested strategies were analysed and compared, in order to evaluate the impact of the different possible approaches on the orientation of the cameras and on the quality of the different generated 3D model. The consistency of the six low-cost action cameras, the impact of a correct estimation of the interior parameters, the quality achievable in the process of tie point extraction and the metric quality of the $3 \mathrm{D}$ models were all considered issues and some first consideration will be reported in the following paragraphs.

\subsection{Cameras consistency}

As is well known low-cost cameras, and sensors in general, can present deformation derived from their mass market production. The six action cameras considered in this research have nominally the same exact specifications. Thus, as demonstrated by the different test performed, each camera has different characteristic and, in particular, one of the cameras presented a set of interior parameters that can lead to an inconsistency of the 360 system considered as a whole. This issue can be negligible if the camera is used as a standalone, as happen in most of the cases, but can be have a negative impact if the sensor is jointly used with the other ones. The different tests performed demonstrated once again the importance of not considering the photogrammetric software as a black box in which operate fully automatic procedures. Also in the most diffused commercial solution, such as Agisoft Photoscan, is possible for the operator to maintain the control of several parameters during the different steps of processing. In particular, in the case of lowcost cameras, and especially if multiple cameras are used together, is crucial for the operator to adopt some best practices. Firstly, during the acquisition phases is important to project and realise a strong network of cameras with a good overlap between them, to facilitate the phases of interior and exterior orientation. A simple intervention of the operator, like the modification of the Exif files, can have a strong impact on the quality of camera calibration and tie point extraction phases that influences also the quality of the generated $3 \mathrm{D}$ model. The use of GCPs and CPs is again really important, not only for scaling 
and georeferencing the model, but also to aid and optimize interior and exterior orientation steps.

Comparing the parameters estimated following the three different approaches (Table 2, Table 3, Table 4), some consideration can be reported. While is clear that following a fully automatic procedure lead to poor results, is important to analyse more in depth how some small interventions can radically change the generated output. Considering the six separate projects as the most reliable, is interesting to notice that the parameters reported in Table 4 can be definitely defined as accurate. The values estimated in the last of the eight projects for the six cameras demonstrate that adopting some best practices on the field and in the post processing phases, allow to calibrate the sensors of the system in a single photogrammetric project.

\subsection{Impact of calibration on image stitching}

In the next sections the importance of a good estimation of camera parameters during the photogrammetric process will be underlined, but the computed parameters can have an impact also in other processes related with the 360 cameras output.

One of the main products derived from these systems is the creation of 360 images or videos, usually represented as an equirectangular projection derived from the stitching of images from multiple source. Nowadays, different software solution exists on the market for the processing of these digital contents, both opensource (Ptgui, Hugin), and commercial (AutoPano Giga, VideoStitch). These software presents common features with the methodological framework of photogrammetry, thus can be subjected to similar issues connected with the sensors specifications. The commercial solution tested in this research is AutoPano Giga (v. 4.2), by Kolor. Using this platform is possible to create spherical images and it works in subsequent step: first of all, SIFT (Scale Invariant Feature Transform) or similar algorithms are used in order to extract tie points from the used images and an RMSe (Root Mean Square error) evaluation is also provided for each calculated tie point. During this process some of the camera parameters are taken into consideration from the software (i.e. focal length, $\mathrm{k} 1, \mathrm{k} 2$ and $\mathrm{k} 3$ coefficient for radial distortion and the coordinates of the principal point); they are partially read from the Exif file and partially extracted from the software database. During this part of the process is possible with some manual editing to modify the information embedded in the Exif file to let the software consider separately the six cameras. In this part of the Autopano workflow the camera calibration parameters extracted in the self-calibration performed in Photoscan were used. Furthermore according to the processing steps the stiching process was realized and the impact on the quality of the process has been evaluate. In Figure 9 an example of some image aberration that were corrected thanks to the lens distortion parameters calculated and imputed for each camera.

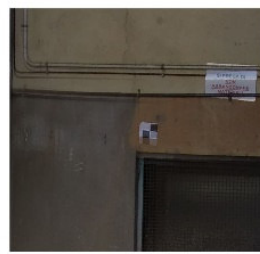

Native Exif

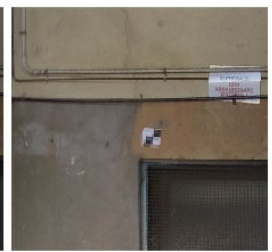

Modified Exif

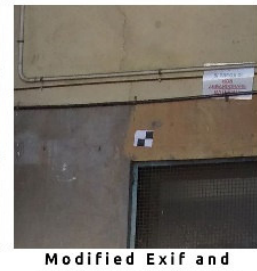
Modified Exif and
camera parameters
Figure 9. Stitching aberration related with the use of different approach for camera interior parameters estimation.

In Figure 13 the parameters used in the stitching software are reported: as in Photoscan using the native Exif the software assumes the same parameters for each camera. Applying again a modification on the Exif is possible to work with separate camera characteristic, but Autopano is not able to estimate the different coefficient with a good level of accuracy. For improving the stitching quality is possible, as is reported before, to use the parameters estimated by the photogrammetric process. There are still some minor issues to fix in the overall stitching of the spherical images, but, as is possible to notice in Figure 9, some major image aberrations can be improved applying the described procedure.

\subsection{Impact of calibration on tie points extraction and 3D model generation}

In order to understand and evaluate the impact of a rigorous and correct calibration of the 360 system on the photogrammetric process, two other analyses were performed. The first analysis is related with an assessment of the quality of the extracted tie points on the two 360 configurations tested: the one automatically processed with the native Exif file and the one processed after the Exif modification. Through a Python script launched by command line it was possible to extract directly from the photogrammetric project generated in Photoscan a .txt file formed by four columns and containing some precious information related with the sparse cloud of tie points. This file contains for each tie point the spatial coordinates and the reprojection error. The points were filtered, excluding the socalled outliners, and all the points with a reprojection error higher than 10 pixels were not considered. The script was applied to the two photogrammetric projects and the obtained data were imported and classified in CloudCompare software, applying a scale of false colours based on the reprojection error of each tie point. An extract of these analyses is reported in Figure 10, where is clearly visible the impact of a correct calibration on the quality of the tie points extracted in the photogrammetric process.
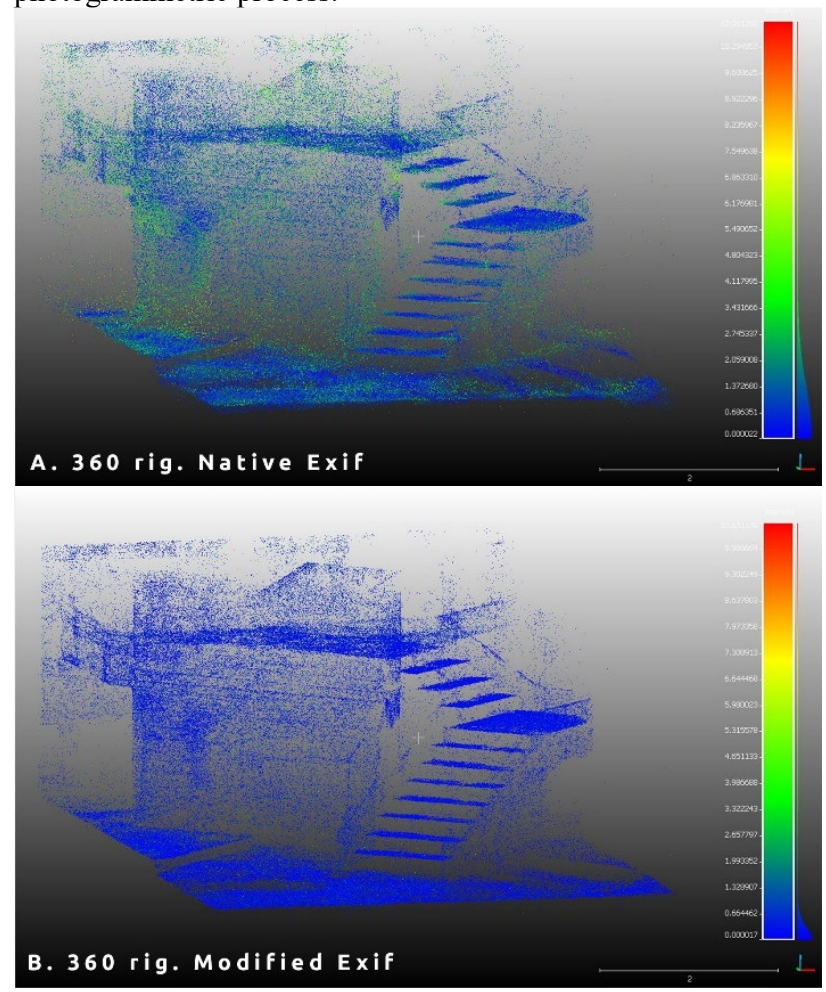

Figure 10. Sparse cloud. Tie point quality based on reprojection error. 360 configuration: automatic process with native Exif (A), process with modified Exif(B) 
In the A configuration is possible to notice how only the $24 \%$ of points presents a reprojection error value minor of 0.5 pixels, while for the B configuration the $72 \%$ of points are comprehend in the same range of values. If we move the observation to the value of 1 pixel of reprojection error the ratio between $\mathrm{A}$ and $\mathrm{B}$ is $40 \%$ to $90 \%$.

\subsubsection{D models validation}

The influence of a good camera calibration on the generation of the 3D model is evident also from a first visual quality assessment between the products derived from the two configurations (Figure 11). To better understand and analyse the overall quality of the generated 3D models some more specific analyses were achieved, using a LiDAR (Light Detection and Ranging) acquisition as ground truth. The laser dataset was acquired using a Faro Focus X120 by CAM2, following the consolidated workflow for acquisition ad post-processing phases, in particular the scans were registered using a cloud to cloud approach and then georeferenced with the same dataset of control points used for the photogrammetric acquisitions. In order to understand how the two different photogrammetric projects were able to correctly represent the geometry of the calibration field, a small sample area was chosen, and the two photogrammetric clouds were compared with the one derived from the laser scanner, using the $\mathrm{C} 2 \mathrm{C}$ (Cloud to Cloud) distances tool implemented in CloudCompare. The results of these analyses are reported in Figure 12: the configuration A resulted in a poor reconstruction of the geometry of the selected portion (only the $4 \%$ of points present a deviation minor of $0.003 \mathrm{~m}$ from the LiDAR cloud) while the B configuration reached good results (the $60 \%$ of points present a deviation minor of $0.003 \mathrm{~m}$ from the LiDAR cloud). Is important to notice that in the A configuration there are also several gaps in the reconstruction of the geometry of the object.

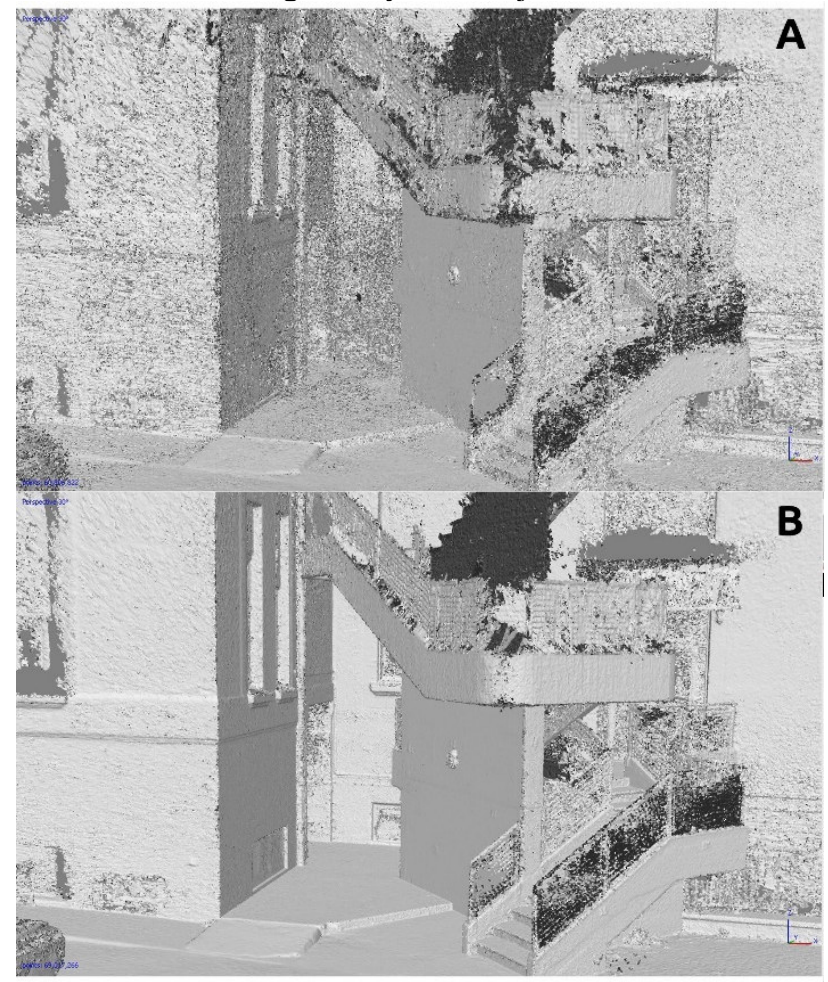

Figure 11. Visual inspection of the Dense Cloud generated in Photoscan. 360 configuration: automatic process with native Exif (A), process with modified Exif(B).
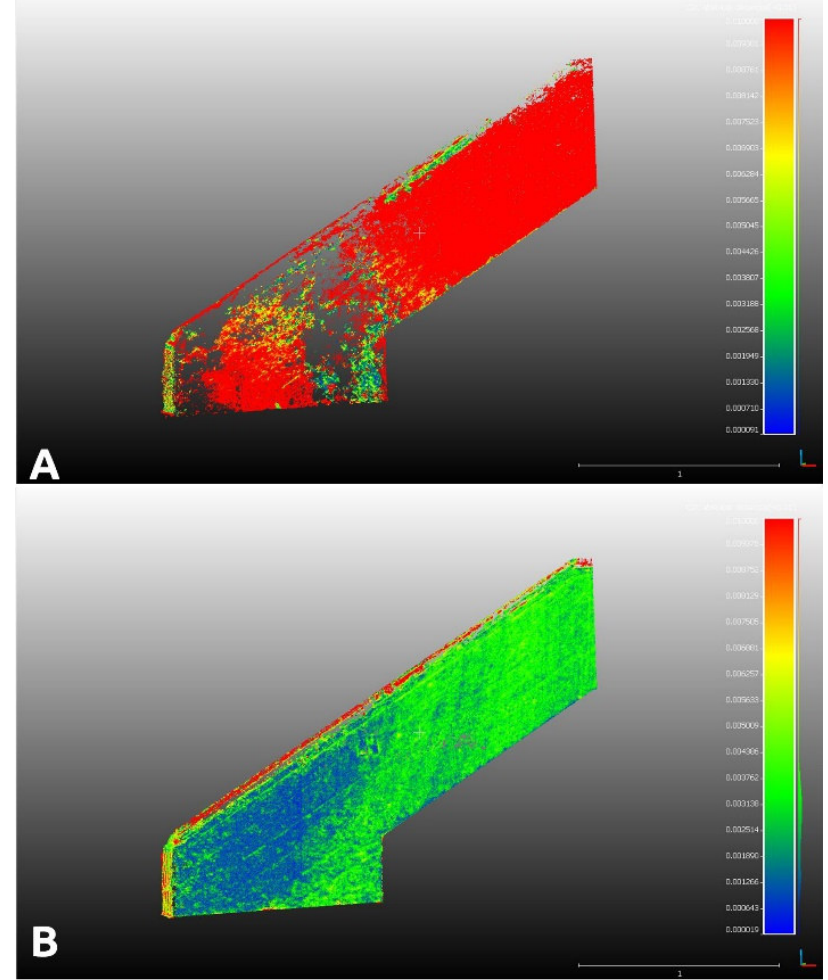

Figure 12. C2C distances analysis performed in CloudCompare with LiDAR data set as ground truth. Max distance set at $0.01 \mathrm{~m}$.

360 configuration: automatic process with native Exif (A), process with modified Exif (B).

\section{DISCUSSION AND FUTURE PERSPECTIVES}

The presented work aimed to evaluate the potentialities of the tested 360 system for metric documentation purposes. Different available configurations of the system were tested both during the acquisition and the photogrammetric process. Starting from a $3 \mathrm{D}$ calibration field carefully projected and using accurate and precise control points, different strategies for the estimation of camera interior orientation parameters were applied. One of the most interesting output of this research can be traced in the possibilities to estimate with a good level of confidence the parameters of the six cameras performing a single acquisition using the 360 rig configuration. The tables reported in the Appendix demonstrate how the intervention of the operator can be crucial in an automated photogrammetric workflow, and that, especially in case of low-cost mass market sensors, the control over these parameters is fundamental. Another important result is the possibility to import the computed parameters of the six cameras in the stitching software solution. The first test performed underline that, also in these preliminary steps of experimentation, an overall improvement of the spherical image produced is appreciable also from a simple visual inspection. Further and more robust analyses need to be performed in order to quantify the impact of these parameters in the stitching process. In the photogrammetric process the impact of the tested practices was assessed with different analyses. Firstly, the reprojection error of each tie points was extracted and analysed for the two 360 configurations. Secondly, a comparison with a more consolidated approach was achieved to evaluate the quality of the processed point clouds in the reconstruction of the object geometry.

Further test must be performed in this direction, a calibration on a different 3D field is ongoing: the aim is to evaluate the performances of the system in a wider environment, widening also the camera-subject acquisition distance; to stress the 
operational features of the six cameras in different contexts. Also, the acquisition in video mode need to be considered and investigated and the use of other photogrammetric software (with the relative calibration models included) must be examined. Finally, the potentialities of the use of spherical images in the photogrammetric process (i.e. spherical photogrammetry) have risen the interest of several researchers: Barazzetti et al., 2017a; Kossieris, Kourounioti, Agrafiotis, \& Georgopoulos, 2018.; Karol Kwiatek \& Tokarczyk, 2015; K Kwiatek \& Tokarczyk, 2014; Ramos \& Prieto, 2016 but a lot of aspects need to be more in depth investigated and evaluate, due to the constant technical evolution of sensors and software available on the market.

\section{REFERENCES}

Balletti, C., Guerra, F., Tsioukas, V., \& Vernier, P. (2014). Calibration of action cameras for photogrammetric purposes. Sensors (Switzerland), 14(9), 17471-17490. https://doi.org/10.3390/s140917471

Barazzetti, L., Previtali, M., \& Roncoroni, F. (2017a). 3D modelling with the samsung gear 360, XLII(March), 1-3. https://doi.org/10.5194/isprsarchives-XLII-2-W3-85-2017

Barazzetti, L., Previtali, M., \& Roncoroni, F. (2017b). Fisheye lenses for 3D modeling: Evaluations and considerations. International Archives of the Photogrammetry, Remote Sensing and Spatial Information Sciences ISPRS Archives, 42(2W3), 79-84. https://doi.org/10.5194/isprs-archivesXLII-2-W3-79-2017

Fangi, G. (2015). Towards an easier orientation for spherical photogrammetry. International Archives of the Photogrammetry, Remote Sensing and Spatial Information Sciences - ISPRS Archives, 40(5W4), 279-283. https://doi.org/10.5194/isprsarchives-XL-5-W4-279-2015

Fangi, G., \& Nardinocchi, C. (2013). Photogrammetric processing of spherical panoramas. Photogrammetric Record, 28(143), 293-311. https://doi.org/10.1111/phor.12031

Fraser, C. S. (1997). Digital camera self-calibration. ISPRS Journal of Photogrammetry and Remote Sensing, 52(4), 149-159. https://doi.org/10.1016/S0924-2716(97)00005-1

Fraser, C. S. (2013). Automatic Camera Calibration in Close Range Photogrammetry. Photogrammetric Engineering \& Remote Sensing, 79(4), 381-388. https://doi.org/10.14358/PERS.79.4.381

Gruen, A., \& Beyer, H. A. (2001). System Calibration Through SelfCalibration. In Calibration and Orientation of Cameras in Computer Vision. (pp. 163-193). Springer, Berlin, Heidelberg. https://doi.org/10.1007/978-3-662-04567-1_7

Holdener, D., Nebiker, S., \& Blaser, S. (2017). Design and Implementation of a Novel Portable $360^{\circ}$ Stereo Camera System With Low-Cost Action Cameras, XLII(November), 28-29.
Kossieris, S., Kourounioti, O., Agrafiotis, P., \& Georgopoulos, A. (2017). Developing a Low-Cost System for 3D Data Acquisition. ISPRS International Archives of the Photogrammetry, Remote Sensing and Spatial Information Sciences, XLII-2/W8(November), 119-126. https://doi.org/10.5194/isprs-archives-XLII-2-W8-119-2017

Kwiatek, K., \& Tokarczyk, R. (2014). Photogrammetric applications of immersive video cameras. https://doi.org/10.5194/isprsannals-II-5-2112014

Kwiatek, K., \& Tokarczyk, R. (2015). Immersive photogrammetry in 3D modelling. Geomatics and Environmental Engineering, 9(2), 51. https://doi.org/10.7494/geom.2015.9.2.51

Läbe, T., \& Förstner, W. (2004). Geometric stability of low-cost digital consumer cameras. Proceedings of the 20th ISPRS Congress, Istanbul, Turkey, 528-535.

Luhmann, T. (2004). A historical review on panorama photogrammetry. International Archives of the Photogrammetry, Remote Sensing and Spatial Information Sciences, 35(5), 8. https://doi.org/10.1.1.101.2588

Luhmann, T., Fraser, C., \& Maas, H. G. (2016). Sensor modelling and camera calibration for close-range photogrammetry. ISPRS Journal of Photogrammetry and Remote Sensing, 115, 37-46. https://doi.org/10.1016/j.isprsjprs.2015.10.006

Markiewicz, J. S., Łapi, S., Bienkowski, R., \& Kaliszewska, A. (2017). The example of using the xiaomi cameras in inventory of monumental objects - first results, XLII(November), 28-29.

Perfetti, L., Polari, C., \& Fassi, F. (2017). Fisheye photogrammetry: Tests and methodologies for the survey of narrow spaces. International Archives of the Photogrammetry, Remote Sensing and Spatial Information Sciences - ISPRS Archives, 42(2W3), 573-580. https://doi.org/10.5194/isprsarchives-XLII-2-W3-573-2017

Ramos, A. P., \& Prieto, G. R. (2016). Only image based for the 3D metric survey of gothic structures by using frame cameras and panoramic cameras. International Archives of the Photogrammetry, Remote Sensing and Spatial Information Sciences - ISPRS Archives, 41(July), 363-370. https://doi.org/10.5194/isprsarchives-XLI-B5-363-2016

Remondino, F., \& Fraser, C. (2006). Digital camera calibration methods: considerations and comparisons. International Archives of Photogrammetry Remote Sensing and Spatial Information Sciences, 36(5), $266-272$.

Schneider, D., Schwalbe, E., \& Maas, H. G. (2009). Validation of geometric models for fisheye lenses. ISPRS Journal of Photogrammetry and Remote Sensing, 64(3), 259-266. https://doi.org/10.1016/j.isprsjprs.2009.01.001

Szeliski, R. (2006). Image Alignment and Stitching. Handbook of Mathematical Models in Computer Vision.

\begin{tabular}{|c|c|c|c|c|c|c|}
\hline \multicolumn{7}{|c|}{ Estimated interior orientation parameters of the six cameras separately considered and processed } \\
\hline & $\mathbf{A}$ & $\mathbf{B}$ & $\mathbf{C}$ & D & $\mathbf{E}$ & $\mathbf{F}$ \\
\hline f(focale -mm) & 3,049 & 3,042 & 3,040 & 3,040 & 3,044 & 3,035 \\
\hline f(focale -px) & 1761,739 & 1757,888 & 1756,620 & 1756,499 & 1758,889 & 1753,511 \\
\hline ex (px) & $-59,928$ & $-76,449$ & 21,457 & $-1,514$ & 48,974 & 50,579 \\
\hline cy (px) & $-23,881$ & $-30,115$ & $-44,285$ & $-6,173$ & 54,031 & 22,681 \\
\hline k1 (mm) & 0,00533 & 0,00523 & 0,00496 & 0,00518 & 0,00518 & 0,00521 \\
\hline k2 (mm) & 0,00018 & 0,00017 & 0,00021 & 0,00020 & 0,00019 & 0,00018 \\
\hline k3 (mm) & $-7,820 \mathrm{E}-06$ & $-7,684 \mathrm{E}-06$ & $-8,792 \mathrm{E}-06$ & $-8,3076 \mathrm{E}-06$ & $-8,068 \mathrm{E}-06$ & $-7,652 \mathrm{E}-06$ \\
\hline b1 (px) & 0,0528 & $-0,4354$ & 0,2083 & $-0,0041$ & $-0,0546$ & $-0,0416$ \\
\hline b2 (px) & $-0,0820$ & $-0,0213$ & 0,0472 & 0,2613 & 0,0571 & 0,0862 \\
\hline p1 (mm) & $-2,640 \mathrm{E}-05$ & $1,6157 \mathrm{E}-05$ & $-8,781 \mathrm{E}-06$ & $2,110 \mathrm{E}-05$ & $1,993 \mathrm{E}-05$ & $5,325 \mathrm{E}-06$ \\
\hline p2 (mm) & $3,470 \mathrm{E}-05$ & $-1,490 \mathrm{E}-07$ & $1,640 \mathrm{E}-06$ & $-1,131 \mathrm{E}-06$ & $-1,470 \mathrm{E}-07$ & $1,667 \mathrm{E}-06$ \\
\hline
\end{tabular}

Table 2. Estimated interior orientation parameters of the six cameras separately considered and processed 


\section{APPENDIX}

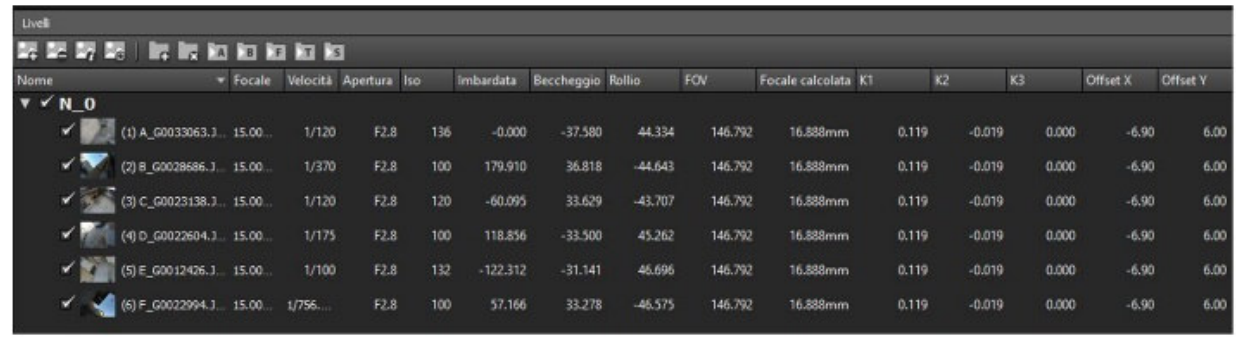

Native Exif

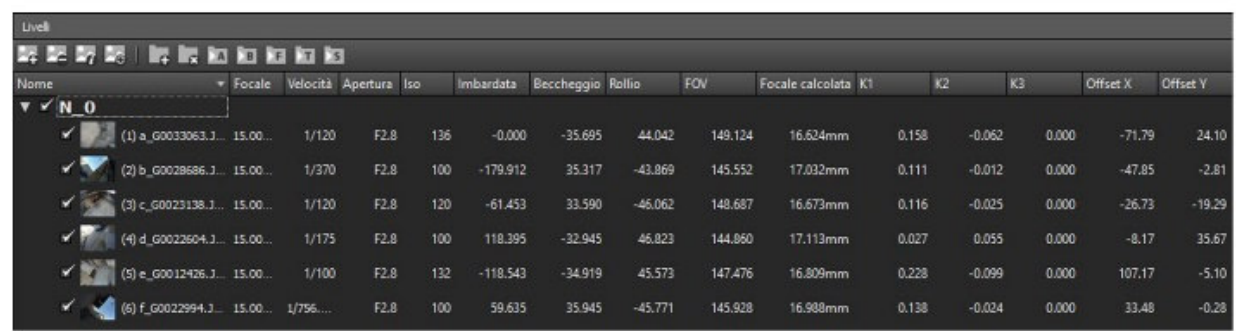

Modified Exif

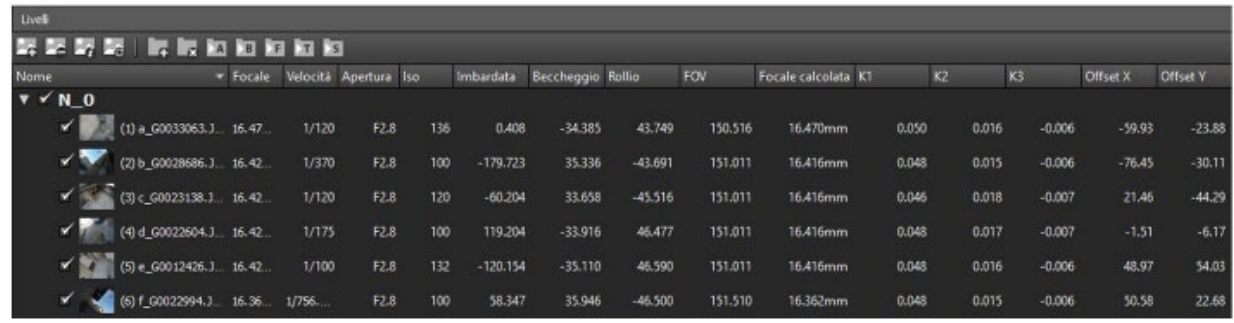

Modified Exif and camera parameters

Figure 13. Camera parameters used in three different approach in AutoPano Giga.

\begin{tabular}{|l|l|}
\hline 360 Rig. Native Exif. Interior orientation parameters \\
\hline $\mathbf{f}($ focale $-\mathbf{m m})$ & 3,038 \\
\hline f(focale $-\mathbf{p x})$ & 1755,593 \\
\hline cx (px) & 0,381 \\
\hline cy (px) & 1,070 \\
\hline $\mathbf{k 1}(\mathbf{m m})$ & 0,00499 \\
\hline $\mathbf{k 2}(\mathbf{m m})$ & 0,00021 \\
\hline $\mathbf{k 3}(\mathbf{m m})$ & $-8,878 \mathrm{E}-06$ \\
\hline b1 (px) & 1,0267 \\
\hline b2 (px) & 0,4841 \\
\hline p1 (mm) & $1,273 \mathrm{E}-05$ \\
\hline $\mathbf{p 2}(\mathbf{m m})$ & $-9,230 \mathrm{E}-07$ \\
\hline
\end{tabular}

Table 3. Estimated interior orientation parameters of the six cameras mounted on the rig and automatically processed with the native Exif

\begin{tabular}{|c|c|c|c|c|c|c|}
\hline \multicolumn{7}{|c|}{360 Rig. Modified Exif. Estimated interior orientation parameters of the six cameras } \\
\hline & $\mathbf{A}$ & B & $\mathbf{C}$ & D & $\mathbf{E}$ & $\mathbf{F}$ \\
\hline f(focale -mm) & 3,050 & 3,038 & 3,040 & 3,041 & 3,045 & 3,035 \\
\hline f(focale -px) & 1762,37711 & 1755,30797 & 1756,610 & 1756,98423 & 1759,15712 & 1753,58122 \\
\hline cx (px) & $-59,740$ & $-76,220$ & 21,893 & $-1,884$ & 48,956 & 50,620 \\
\hline cy (px) & $-24,235$ & $-30,213$ & $-44,387$ & $-6,310$ & 54,120 & 22,333 \\
\hline k1 (mm) & 0,00526 & 0,00620 & 0,00503 & 0,00516 & 0,00512 & 0,00521 \\
\hline k2 (mm) & 0,00019 & 0,00001 & 0,00020 & 0,00020 & 0,00019 & 0,00018 \\
\hline $\mathrm{k3}(\mathrm{mm})$ & $\begin{array}{l}-0,00001 \\
\end{array}$ & 0,00000 & $\begin{array}{l}-0,00001 \\
\end{array}$ & $-0,00001$ & $\begin{array}{l}-0,00001 \\
\end{array}$ & $\begin{array}{l}-0,00001 \\
\end{array}$ \\
\hline b1 (px) & $-0,1357$ & $-0,1955$ & 0,1670 & $-0,2115$ & $-0,0670$ & 0,0277 \\
\hline b2 (px) & $-0,0682$ & $-0,2481$ & $-0,0361$ & 0,2873 & 0,1400 & $-0,0095$ \\
\hline p1 (mm) & $\begin{array}{l}-4,89731 \mathrm{E}-07 \\
\end{array}$ & $1,33023 \mathrm{E}-05$ & $-1,009 \mathrm{E}-05$ & $2,237 \mathrm{E}-05$ & $2,316 \mathrm{E}-05$ & $1,340 \mathrm{E}-06$ \\
\hline p2 (mm) & $4,20493 \mathrm{E}-07$ & $4,505 \mathrm{E}-07$ & $1,868 \mathrm{E}-06$ & $-9,839 \mathrm{E}-07$ & $-2,416 \mathrm{E}-07$ & $2,162 \mathrm{E}-06$ \\
\hline
\end{tabular}

Table 4. Estimated interior orientation parameters of the six cameras mounted on the rig and processed after the Exif modification

\footnotetext{
* Corresponding author
} 